\title{
Ultrasonographic guidance for spinal extracranial radiosurgery: technique and application for metastatic spinal lesions
}

\author{
Timothy C. Ryken, M.D., Sanford L. Meeks, Ph.D., Vincent Traynelis, M.D., \\ John Haller, Ph.D., Lionel G. Bouchet, Ph.D., Francis J. Bova, Ph.D., \\ Edward C. Pennington, M.S., and John M. Buatti, M.D.
}

Departments of Neurosurgery, Radiation Oncology, and Radiology, University of Iowa, Iowa City, Iowa; and Department of Neurological Surgery, University of Florida, Gainesville, Florida

\begin{abstract}
Object. The relatively stationary anatomy of the intracranial compartment has allowed the development of stereotactic radiosurgery as an effective treatment option for many intracranial lesions. Difficulty in accurately tracking extracranial targets has limited its development in the treatment of these lesions. The ability to track extracranial structures in real time with ultrasound images allows a system to upgrade and interface pretreatment volumetric images for extracranial applications. In this report the authors describe this technique as applied to the treatment of localized metastatic spinal disease.

Methods. The extracranial stereotactic system consists of an optically tracked ultrasonography unit that can be registered to a linear accelerator coordinate system. Stereotactic ultrasound images are acquired following patient positioning, based on a pretreatment computerized tomography (CT) simulation. The soft-tissue shifts between the virtual CT-based treatment plan and the actual treatment are determined. The degree of patient offset is tracked and used to correct the treatment plan.

The ultrasonography-based stereotactic navigation system is accurate to within an approximate means of $1.5 \mathrm{~mm}$ based on testing with an absolute coordinate phantom. A radiosurgical treatment was delivered using the system for localization of a metastatic spinal lesion. Compared with the virtual CT simulation, the actual treatment plan isocenter was shifted $12.2 \mathrm{~mm}$ based on the stereotactic ultrasound image. The patient was treated using noncoplanar beams to a dose of $15.0 \mathrm{~Gy}$ to the $80 \%$ isodose shell in a single fraction.

Conclusions. A system for high-precision radiosurgical treatment of metastatic spinal tumors has been developed, tested, and applied clinically. Optical tracking of the ultrasonography probe provides real-time tracking of the patient anatomy and allows computation of the target displacement prior to treatment delivery. The results reported here suggest the feasibility and safety of the technique.
\end{abstract}

\section{KEY WORDS • extracranial stereotactic radiosurgery • three-dimensional ultrasonography • neuronavigation $\bullet$ optical tracking $\bullet$ metastasis $\bullet$ spine}

In an attempt to replace an open surgical approach, intracranial stereotactic radiosurgery has been developed as a single fraction of irradiation directed to an intracranial target in selected patients. As originally described, a stereotactic head frame is attached to the patient, providing a rigid frame of reference for image acquisition and target localization. Image coordinates referable to the head frame are transformed into stereotactic coordinates based on the location of the frame and patient anatomy. The re-

Abbreviations used in this paper: $\mathrm{AP}=$ anteroposterior; $\mathrm{CT}=$ computerized tomography; LED = light-emitting diode; LINAC = linear accelerator; PITV = prescription isodose to tumor volume; $\mathrm{PTV}=$ planning target volume $; 2 \mathrm{D}=$ two-dimensional $3 \mathrm{D}=$ threedimensional; $\mathrm{VB}=$ vertebral body. sultant 3D datasets allow precision planning for radiosurgery or image-guided surgical procedures. The rigid localization of this system combined with the relatively fixed intracranial anatomy allow the accurate targeting of radiation and the calculation of a dose distribution that conforms accurately to the target volume. Additional advantages of this minimally invasive treatment for brain tumors include the single outpatient treatment paradigm and the limited exposure of normal brain tissue to radiation.

Despite these advantages, the application of radiosurgery to extracranial structures has been limited by the requirement of rigid stereotactic fixation. In some cases standard head ring localization has been used to treat tumors confined to the base of skull and upper cervical spine, ${ }^{2,6-12,22}$ and alternative methods have been proposed 
to extend stereotactic localization to extracranial targets. ${ }^{13-15,17,24,25,28}$ The challenge has been to ensure accurate and precise extracranial delivery of radiation because the target position can shift relative to bone anatomy between the time of image acquisition and that of treatment, as well as during the treatment. Progress has been made in applying real-time imaging to improve the accuracy and precision of radiotherapy localization, 1,16,19,27 including the use of ultrasonography as described previously by our group. ${ }^{3}$ In this report we describe the initial clinical application of a system for the radiosurgical treatment of extracranial metastatic spinal tumors in which optically guided ultrasonography is used.

\section{CLINICAL MATERIAL AND METHODS}

\section{Optically Guided Ultrasonography for Extracranial Radiosurgery}

Three-dimensional treatment planning in image-guided therapy requires acquisition of $3 \mathrm{D}$ volumetric image sets, such as CT scanning, magnetic resonance imaging, or positron emission tomography studies, relative to a patient-specific fiducial system. A virtual simulation of a patient's treatment is created using the volumetric image data. The placement of radiation beams is modeled on the virtual patient, and radiation dose distributions are calculated and displayed on the images. We have previously described a system for noninvasive localization of intracranial targets based on optical tracking of a dental tray attached to the patient's maxillary dentition., ${ }^{4,5,18,20} \mathrm{Al}$ though this system was originally designed for fractionated stereotactic radiotherapy, it has proven to provide extremely accurate localization relative to the treatment room and has therefore been used for single-fraction radiosurgery in a selected cohort of patients.

This procedure has limited applicability to extracranial structures because of the tendency of outside soft-tissue targets to move relative to rigid fixation points (for example, bone structures) between the times of image acquisition, treatment planning, and treatment delivery. Realtime imaging allows the position of the target to be upgraded and tracked during the subsequent treatment delivery. With this in mind, we developed a system to integrate 3D ultrasonography (SonArray, ZMed, Inc., Ashland, MA) to correct for these misalignments at the time of treatment. Ultrasonography is a flexible and inexpensive imaging modality adaptable for use in surgical planning or radiation therapy delivery. The acquisition and interpretation of ultrasound images can be technically challenging and is highly dependent on the skill and expertise of the operator in manipulating the transducer and mentally transforming the 2D images into a $3 \mathrm{D}$ tissue structure. Three-dimensional ultrasonography overcomes this limitation, but current systems are expensive. We have developed a technique to generate 3D ultrasonography datasets through optical tracking of free handacquired 2D ultrasound images. The ultrasonography probe is guided over the anatomical region of interest to acquire a 2D ultrasound image set that is transferred to a computer workstation by using a standard video link. The position and angulation of the ultrasonography probe is determined using an array of four infrared LEDs attached

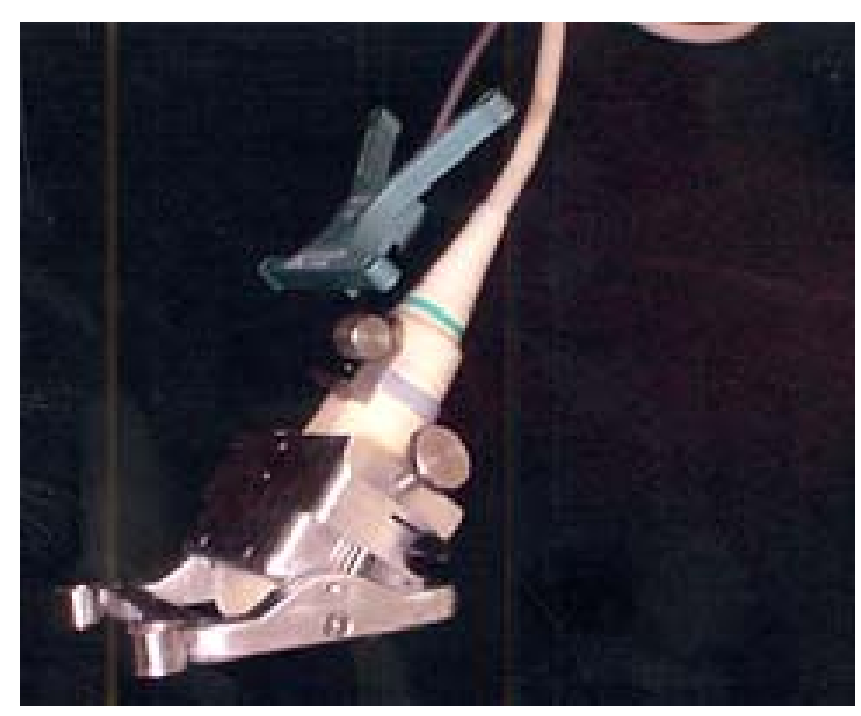

Fig. 1. The ultrasonography probe is tracked via an array of four infrared LEDs rigidly attached to it. A rocking cradle is used to help guide the free-hand acquisition and to assure that no gaps exist in the final ultrasound image volume.

to the probe (Fig. 1). Similar to our system for intracranial optic guidance, charged-coupled device cameras are used to determine the positions of the infrared LEDs, and this information is put into the computer workstation. The position of each image plane can therefore be determined using the infrared LEDs, and an ultrasound-based volume can be reconstructed by coupling the position information with the image (Fig. 2).

In addition to building the 3D image volume, image optic guidance is used to determine the absolute position of the ultrasound image volume in the treatment room coordinate system. Because the relative position of the 3D image volume and the ultrasound is fixed, the knowledge of the probe position in the treatment room coordinate system at the time of image acquisition is sufficient to determine the position of the image volume relative to the LINAC isocenter. The determination of the relative position of the image and probe corresponds to a calibration step that is performed at the time at which the system is installed. ${ }^{3}$

Preclinical Testing. The accuracy of optically guided 3D ultrasonography for patient positioning was tested using a specially designed absolute ultrasonography phantom. ${ }^{3}$ This phantom consists of 15 echoic spheres embedded in a tissue-equivalent nonechoic medium. The spheres are arranged in sets of five located at three different depths: 30,60 , and $110 \mathrm{~mm}$. The phantom was precisely manufactured so that the room locations of the spheres are known to within $0.2 \mathrm{~mm}$. A CT scan $(0.51 \times 0.51 \times 1.25$ $\mathrm{mm}$ ) of the phantom was acquired in which a passive infrared tracking array was attached to it. An infrared array was used to track the position of the phantom in the room coordinate system. The ultrasonography phantom was then placed in the treatment vault and the ultrasonography probe was fixed on top of the phantom. Coupling of the probe and the phantom was achieved using a thin layer 


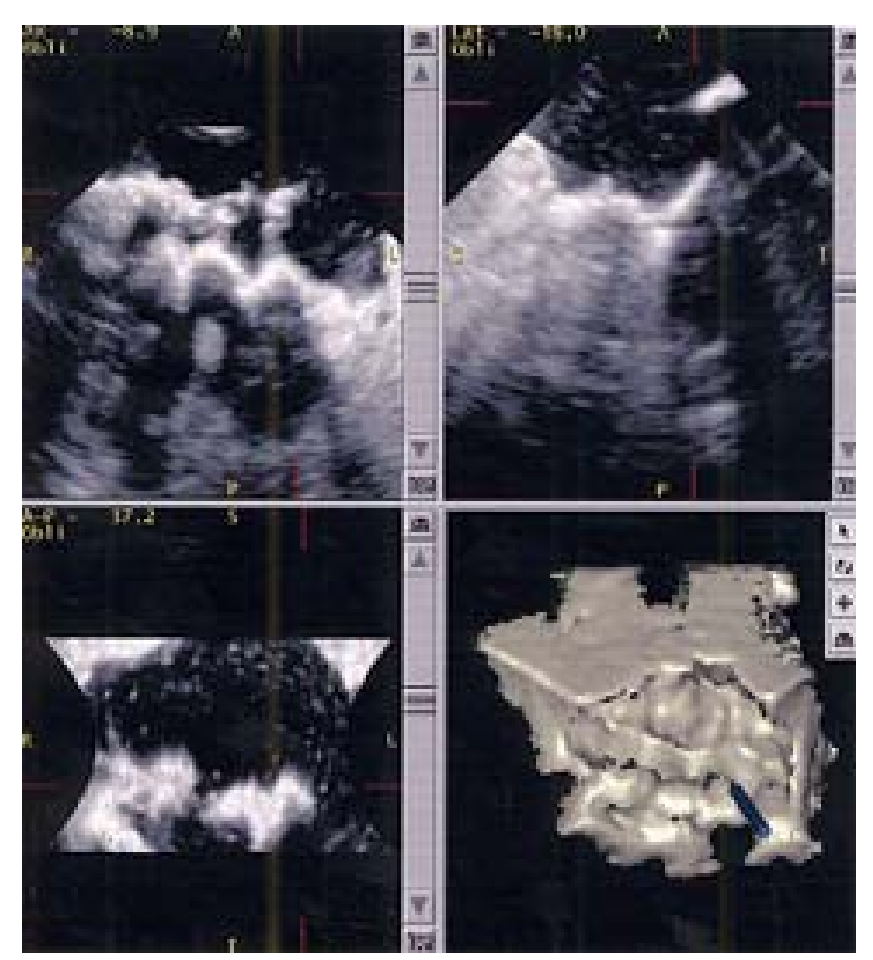

Fig. 2. Three-dimensional ultrasound images (upper left, upper right, and lower left) of VBs demonstrating three orthogonal views along with a $3 \mathrm{D}$ reconstruction (lower right).

of water. Next, a sphere within the phantom was selected as the target sphere and positioned at the room isocenter based on the CT optical tracking of the phantom. The localization of the target sphere was determined using our $3 \mathrm{D}$ ultrasonography guidance system. The $3 \mathrm{D}$ volume of the sphere was acquired ultrasonographically by using optical tracking as described previously. The 3D ultrasonography-based position of the target sphere was determined by finding the center of the sphere in the image by using a circle tool placed on each three-orthogonal ultrasound view. The accuracy of the target localization demonstrated using the 3D ultrasonography optically guided system was thus determined by comparing its experimentally determined position to the known position of the target sphere.

Clinical Application. Clinical use of ultrasound image guidance follows the following sequence. Prior to CT scanning, the patient is immobilized in a customized vacuum pillow that is commonly used in radiation therapy. The CT scan is acquired with the patient immobilized in the same position that will be used during the radiotherapy, to maintain a generally consistent position of mobile anatomy. In addition, a passive array, such as that described for the intracranial optically guided system, is attached to the immobilization device. The CT scans are transferred to our 3D treatment planning system (Pinnacle3; ADAC, Milipitas, CA), where the tumor volume and normal structures of interest are delineated. These contours determine the 3D target volume for treatment planning as well as normal tissue volumes for plan evaluation. A treatment plan is then designed to conform the prescription dose closely to the planning target volume, while minimizing the dose to the nearby normal structures. This is achieved using maximally separated noncoplanar beams. Maximal beam separation provides unique beam entry and exit pathways for each beam, thereby optimizing the dose gradient outside of the target volume. In addition, maximal beam separation provides the most unique beam's-eye-views of the target volume, thereby maximizing the plan conformality. This is an extension of geometric planning techniques used in intracranial stereotactic radiotherapy. ${ }^{26}$ Each beam is shaped to match the beam's-eye-view projection of the planning target volume by using a multileaf collimator that has a 5-mm leaf resolution at isocenter (Millennium MLC120; Varian Oncology Systems, Palo Alto, CA). The field shapes are designed with zero margin added to the beam's-eye-view projection of the planning target volume and multileaf collimator leaves at the edge of the target.

The day of the treatment, the patient is placed in the same immobilization cushion that was used for obtaining CT scans. The patient is moved to the isocenter based on tracking of the infrared reflective array attached to the immobilization device. A 3D ultrasound image set is then acquired and reconstructed in the computer workstation. The target volume and critical structure outlines, as delineated on the planning CT scans, are then overlaid on the ultrasound volume. The contours are then manipulated until they align perfectly with the anatomical structures on the ultrasound images. The amount of movement required to align the contours with the ultrasound images determines the magnitude of the target misregistration with isocenter. The target is then placed at the isocenter by tracking the infrared array attached to the patient immobilization system. After registering the target position with the LINAC isocenter, orthogonal port films (generally AP and lateral) are obtained and compared with digitally reconstructed radiographs obtained during the planning process. Once all of the setup information has been verified, treatment proceeds as planned.

\section{Clinical Case Report}

Our initial case is a 55-year-old man in whom a Stage IA squamous cell carcinoma of the right upper lobe was originally diagnosed and who underwent resection of the right upper lobe and middle lobe in March 1997. Three years later he experienced increased cough, facial fullness, and dyspnea, and CT scanning subsequently revealed a mediastinal mass compressing the superior vena cava. In the summer of 2000 examination of a needle biopsy specimen demonstrated metastatic squamous cell carcinoma, for which he underwent radiotherapy $(66.6 \mathrm{~Gy}$ in 37 fractions) and carbo Taxol chemotherapy concurrently. In December 2000, follow-up CT scanning demonstrated a $2.5 \times 4 \times 5-\mathrm{cm}$ right paravertebral mass at $\mathrm{T}-11$. This was treated with taxotere chemotherapy-four monthly cycles followed by four weekly cycles, the last being administered in May 2001. Follow-up CT scanning of the chest demonstrated slight enlargement of the paravertebral mass, now with invasion into the VB. He was referred to the radiation oncology department in June 2001, after experiencing progressive back pain for 1 month. 
TABLE 1

Summary of distances in the AP, lateral, and axial directions determined ultrasonographically*

\begin{tabular}{cccc}
\hline \hline & \multicolumn{3}{c}{ Distance $(\mathrm{mm})$} \\
\cline { 2 - 4 } Depth $(\mathrm{mm})$ & AP & Lat & Axial \\
\hline 30 & $1.8 \pm 0.3$ & $0.8 \pm 0.4$ & $0.3 \pm 0.2$ \\
60 & $1.1 \pm 0.3$ & $0.4 \pm 0.3$ & $0.2 \pm 0.2$ \\
110 & $1.3 \pm 0.6$ & $0.6 \pm 0.4$ & $0.6 \pm 0.3$ \\
all & $1.4 \pm 0.5$ & $0.6 \pm 0.4$ & $0.4 \pm 0.3$ \\
\hline
\end{tabular}

* All values are presented as the means \pm standard deviations.

\section{RESULTS}

\section{Absolute Accuracy of 3D Ultrasonography Guidance}

As described previously, the room positions of the 15 spheres in the absolute phantom were determined experimentally using the 3D ultrasonography system and compared against their known positions. In averaging the difference between the measured and expected among all 15 spheres at all depths, the full accuracy of this system for patient positioning was found to be on average $1.4 \mathrm{~mm}$ in AP, $0.6 \mathrm{~mm}$ in lateral, and $0.4 \mathrm{~mm}$ in axial projections. Although the measurements were consistent, there was a small degradation in accuracy with increasing depth. Over all depths, however, the accuracy of target repositioning using our ultrasound image-guided system was maintained below approximately $1.5 \mathrm{~mm}$ (Table 1 ).

\section{Patient Treatment}

The patient was immobilized in a customized vacuum cushion, and a volumetric CT scan was acquired with $1 \times$ $1 \times 2-\mathrm{mm}$ resolution. The images were transferred to the Pinnacle treatment planning system, where the PTV, spinal cord, and VBs were delineated. A treatment plan was generated using nine noncoplanar beams that maintained maximal beam separation while minimizing the dose to the spinal cord (Fig. 3). Because the patient previously underwent full-dose radiotherapy, it was imperative to spare the spinal cord. The resultant treatment plan (Fig. 4) was prescribed to deliver 15.0 Gy to the $80 \%$ isodose volume (normalized to the maximum dose) in a single fraction. The appropriateness of the treatment plan was evaluated using the PITV, which is a metric that describes the plan conformality. The PITV equals 1 for an "ideal" plan in which the prescription isodose exactly matches the PTV, and an acceptable intracranial radiosurgery plan is defined as a plan with PITV greater than $2 .{ }^{23}$ In our case, the PITV was calculated as 1.53 .

The images and treatment plan data were then transferred to the SonArray station. The patient was placed on the treatment couch and was initially positioned using the optically-guided system tracking the reference array attached to the patient's immobilization device. A 3D volume was determined ultrasonographically, and the contours of the tumor, spinal cord, and VBs, as determined on the planning CT scan, were overlaid on the ultrasound image volume. The outlines were manipulated until the best fit between the contours and the ultrasound images was obtained (Fig. 5). Treatment proceeded as planned. The patient experienced no significant acute sequelae of the treatment.

\section{DISCUSSION}

Radiosurgery was initially conceived as a method for creating neurosurgical intracranial lesions. Although the treatment paradigm requires extreme precision, radiosurgery has dramatically changed the treatment for many central nervous tumors. Our initial experience using a frameless technique for cranial radiosurgery has been previously described. ${ }^{21}$ Because of the need for extreme precision, radiosurgery has been restricted primarily to intracranial applications. Extracranial application of highprecision radiosurgery is more complex because motion of soft-tissue targets between the time of image acquisition and treatment delivery is unavoidable. We have developed a system that relies on ultrasonographic guidance to correct precisely for internal organ motion and adjust the position of the target at the isocenter of the treatment. Because our image-guided system uses reconstructed 3D images, it has the ability to quantify the patient's alignment in six degrees of freedom. Furthermore, because the ultrasound image set is optically tracked, patient positioning feedback can be provided in real time, allowing the target position to be monitored intraoperatively. This method provides easy and precise feedback regarding the patient position based on image localization, which is very similar to optical systems already used for intracranial targets.

The tracking accuracy of our 3D ultrasound imageguided system was measured to be approximately $1.5 \mathrm{~mm}$, therefore demonstrating the potential of this technique for precision patient positioning. It is important to note that these measurements represent the overall accuracy, and the system integrates all the steps required for its use in a radiotherapy environment (resolution of CT scans, ultrasonography tracking accuracy, and optical tracking of CTdetermined volume for repositioning).

Our clinical observations in our initial case treated with extracranial radiosurgery demonstrate the feasibility and

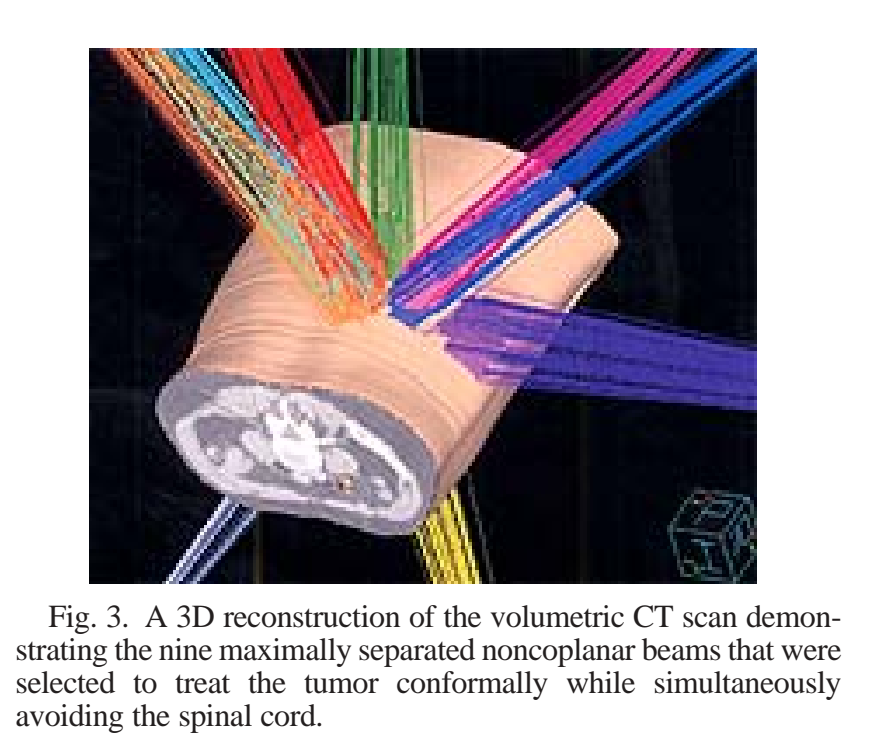



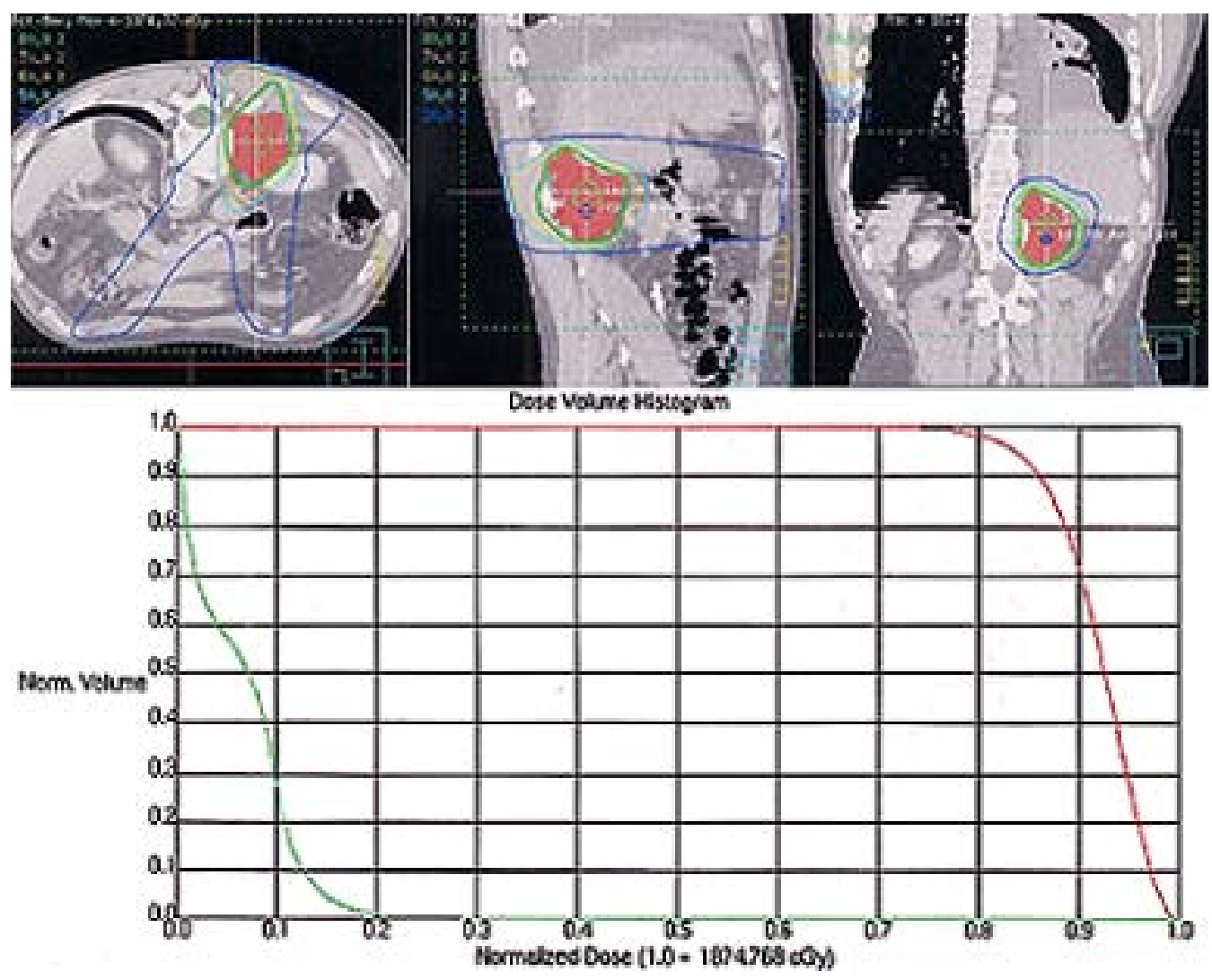

Fig. 4. Upper: The treatment isodose distribution is shown superimposed on axial (left), sagittal (center), and coronal (right) CT scans through the center of the treatment volume, which is shown as a red colorwash. The thick green isodose line surrounding the PTV is the treatment line ( $15 \mathrm{~Gy}$ to the $80 \%$ isodose), with the $70,60,50$, and $20 \%$ isodose lines also shown. Lower: Dose volume histogram for the PTV (red) and spinal cord (green) showing that greater than $97 \%$ of the PTV receives the prescription dose, and the maximum spinal cord dose is approximately $20 \%$ of maximum (3.8 Gy).
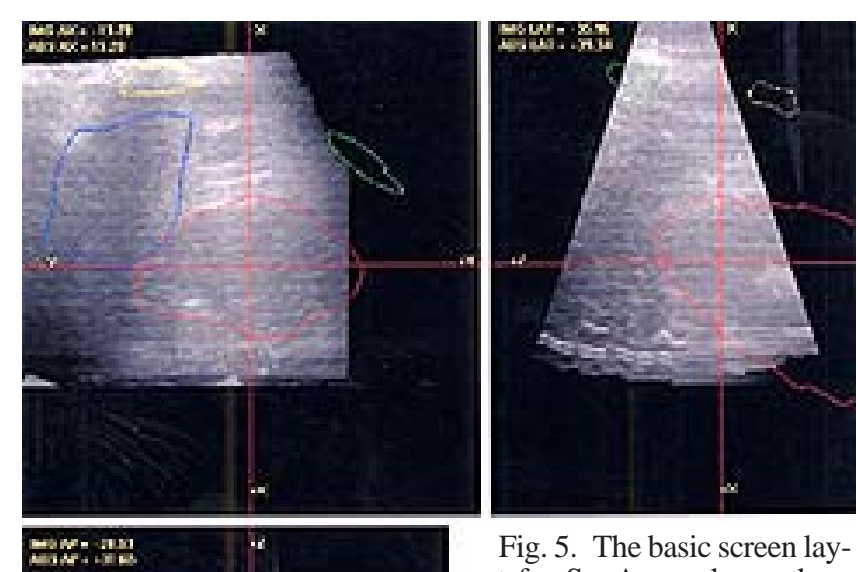

Fig. 5. The basic screen layout for SonArray shows three orthogonal views through the ultrasound image volume with the contours from the planning CT scan (PTV is red) superimposed. By manipulating the contours until they align with the ultrasound images, any patient misalignment can be detected and corrected. initial safety of this approach. We believe this represents the first report of a noninvasive ultrasonography-guided technique for the delivery of single-fraction radiosurgical treatment to a paraspinal target. The degree of accuracy achieved using this technique is acceptable in properly selected patients and provides a noninvasive treatment alternative with a high level of patient satisfaction. The long-term outcome of this approach is unknown. Further investigation is required, but this procedure, in which a single, high-dose treatment is used, offers the potential for rapid, effective palliation and low morbidity. The application of this system not only has potential to treat patients who have previously undergone surgery and radiotherapy but also can serve as an adjuvant to conventional radiotherapy. As has been demonstrated in cases of intracranial radiosurgery, extracranial stereotactic radiotherapy may offer an alternative initial treatment for patients with focal metastatic spinal disease.

\section{Disclosure}

Development of the ultrasonography navigational system was performed in collaboration with ZMed, Inc.

\section{References}

1. Adler JR Jr, Chang SD, Murphy MJ, et al: The Cyberknife: a 
frameless robotic system for radiosurgery. Stereotact Funct Neurosurg 69:124-128, 1997

2. Bajada C, Selch M, De Salles A, et al: Application of stereotactic radiosurgery to the head and neck region. Acta Neurochir Suppl 62:114-117, 1994

3. Bouchet LG, Meeks SL, Goodchild G, et al: Calibration of three-dimensional ultrasound images for image-guided radiation therapy. Phys Med Biol 46:559-577, 2001

4. Bova FJ, Buatti JM, Friedman WA, et al: The University of Florida frameless high-precision stereotactic radiotherapy system. Int J Radiat Oncol Biol Phys 38:875-882, 1997

5. Buatti JM, Bova FJ, Friedman WA, et al: Preliminary experience with frameless stereotactic radiotherapy. Int J Radiat Oncol Biol Phys 42:591-599, 1998

6. Buatti JM, Friedman WA, Bova FJ, et al: Linac radiosurgery for locally recurrent nasopharyngeal carcinoma: rationale and technique. Head Neck 17:14-19, 1995

7. Chua DT, Sham JS, Hung KN, et al: Stereotactic radiosurgery as a salvage treatment for locally persistent and recurrent nasopharyngeal carcinoma. Head Neck 21:620-626, 1999

8. Cmelak AJ, Cox RS, Adler JR, et al: Radiosurgery for skull base malignancies and nasopharyngeal carcinoma. Int J Radiat Oncol Biol Phys 37:997-1003, 1997

9. Firlik KS, Kondziolka D, Lunsford LD, et al: Radiosurgery for recurrent cranial base cancer arising from the head and neck. Head Neck 18:160-166, 1996

10. Kaplan ID, Adler JR, Hicks WL Jr, et al: Radiosurgery for palliation of base of skull recurrences from head and neck cancers. Cancer 70:1980-1984, 1992

11. Kocher M, Voges J, Staar S, et al: Linear accelerator radiosurgery for recurrent malignant tumors of the skull base. Am J Clin Oncol 21:18-22, 1998

12. Kondziolka D, Lunsford LD: Stereotactic radiosurgery for squamous cell carcinoma of the nasopharynx. Laryngoscope 101:519-522, 1991

13. Hamilton AJ, Lulu BA: A prototype device for linear accelerator-based extracranial radiosurgery. Acta Neurochir Suppl 63: 40-43, 1995

14. Hamilton AJ, Lulu BA, Fosmire H, et al: LINAC-based spinal stereotactic radiosurgery. Stereotact Funct Neurosurg 66: $1-9,1996$

15. Hamilton AJ, Lulu BA, Fosmire H, et al: Preliminary clinical experience with linear accelerator-based spinal stereotactic radiosurgery. Neurosurgery 36:311-319, 1995

16. Lattanzi J, McNeeley S, Hanlon A, et al: Ultrasound-based stereotactic guidance of precision conformal external beam radiation therapy in clinically localized prostate cancer. Urology 55: 73-78, 2000
17. Lohr F, Debus J, Frank C, et al: Noninvasive patient fixation for extracranial stereotactic radiotherapy. Int J Radiat Oncol Biol Phys 45:521-527, 1999

18. Meeks SL, Bova FJ, Wagner TH, et al: Image localization for frameless stereotactic radiotherapy. Int J Radiat Oncol Biol Phys 46:1291-1299, 2000

19. Murphy MJ, Adler JR Jr, Bodduluri M, et al: Image-guided radiosurgery for the spine and pancreas. Comput Aided Surg 5: 278-288, 2000

20. Phillips MH, Singer K, Miller E, et al: Commissioning an image-guided localization system for radiotherapy. Int J Radiat Oncol Biol Phys 48:267-276, 2000

21. Ryken TC, Meeks SL, Pennington EC, et al: Initial clinical experience with frameless stereotactic radiosurgery: analysis of accuracy and feasibility. Int J Radiat Oncol Biol Phys 51: 1152-1158, 2001

22. Samblas JM, Bustos JC, Gutierrez-Diaz JA, et al: Stereotactic radiosurgery of the foramen magnum region and upper neck lesions: technique modification. Neurol Res 16:81-82, 1994

23. Shaw E, Kline R, Gillin M, et al: Radiation Therapy Oncology Group: radiosurgery quality assurance guidelines. Int J Radiat Oncol Biol Phys 27:1231-1239, 1993

24. Takacs I, Hamilton AJ: Extracranial stereotactic radiosurgery: applications for the spine and beyond. Neurosurg Clin N Am 10:257-270, 1999

25. Takacs I, Hamilton AJ, Lulu B, et al: Frame based stereotactic spinal radiosurgery: experience from the first 19 patients treated. Stereotact Funct Neurosurg 73:69, 1999

26. Tome WA, Meeks SL, Buatti JM, et al: A high-precision system for conformal intracranial radiotherapy. Int J Radiat Oncol Biol Phys 47:1137-1143, 2000

27. Troccaz J, Laieb N, Vassal P, et al: Patient setup optimization for external conformal radiotherapy. J Image Guid Surg 1: 113-120, 1995

28. Wang TL, Solberg TD, Medin PM, et al: Infrared patient positioning for stereotactic radiosurgery of extracranial tumors. Comput Biol Med 31:101-111, 2001

Manuscript received October 19, 2001.

Accepted in final form November 2, 2001.

Funding for this work was provided by Whitaker Foundation Biomedical Engineering Research Grant No. RG-98-0278.

Address reprint requests to: Timothy C. Ryken, M.D., Department of Neurosurgery, University of Iowa, 200 Hawkins Drive, Iowa City, Iowa 52242-1077. email: timothy-ryken@uiowa.edu. 